\title{
Responses to vaccinations vary after rituximab therapy
}

Patients with rheumatoid arthritis (RA) have diminished immune responses as a result of the factors involved in the pathogenesis of the disease itself and the the immunosuppressive agents often used to treat it. Vaccinations against common infections, therefore, are an important therapeutic goal for these patients. The results of two controlled trials, now published in the January 2010 issue of Arthritis and Rheumatism, suggest that treatment with the B-cell depleting agent, rituximab, lowers the response to certain vaccines, but not to others.

Bingham and colleagues investigated immune responses in 103 patients with RA who were randomly assigned to receive rituximab and methotrexate or methotrexate alone; all patients also received tetanus toxoid and pneumococcal polysaccharide vaccines. The proportion of positive, T-cell dependent, responses to the tetanus vaccine was similar in both groups $-39.1 \%$ of rituximab-treated patients had a 4 -fold or greater increase in anti-tetanus immunoglobulin $\mathrm{G}$ titers from baseline compared with $42.3 \%$ of the methotrexate-only group. By contrast, responses to the polysaccharide pneumococcal vaccine, which are T-cell independent, were markedly reduced in patient receiving rituximab compared with methotrexate-just $57 \%$ of the former showed a positive result compared with $82 \%$ of the latter.

Owing to seasonal variability of the influenza vaccine, Bingham and colleagues were unable to examine the potential effects of rituximab on immune responses to the influenza vaccine. This topic was addressed in a small trial by van Assen et al. published in the same issue of Arthritis and Rheumatism. In the study, rituximab-treated patients $(n=23)$ had drastically lower geometric mean titers of antibodies for three strains of influenza (A/H3N2 strain, A/H1N1 strain and B strain) compared with methotrexatetreated patients and healthy controls.

Notably, however, patients receiving rituximab who were vaccinated 6-10 months after therapy showed a slightly better response than those who were vaccinated sooner (4-8 weeks after therapy). As the B-cell population begins to recover at around 6-9 months after depletion, vaccines such as influenza that rely on a B-cell response might be more effective if given at a later date following therapy. Interestingly, van Assen et al. also found that rituximab-treated patients who had received a prior influenza vaccine had an enhanced immune response compared with those who had not previously been vaccinated.

Thomas Dörner from the Department of Medicine, Rheumatology and Clinical Immunology of the Charite Hospital in Berlin, Germany, remarks that these studies "confirm very nicely the impairments in the response to vaccines in RA, and in particular highlight the need for immunizations in our patients, independent of their disease stage". Dörner believes, however, that "In terms of vaccination under rituximab therapy most aspects of immediate vaccine responses have been comprehensively covered. We now need long-term data on the stability of protective immunoglobulin titers, and comparative data for other, widely used therapies for RA".

Negin Nassabeh

Original articles Bingham, C. 0. et al. Immunization
responses in rheumatoid arthritis patients treated with
rituximab. Arthritis Rheum. 62,64-74 (2010) | van Assen, S.
et al. Humoral responses after influenza vaccination are
severely reduced in patients with rheumatoid arthritis treated
with rituximab. Arthritis Rheum. 62, 75-81 (2010)

